The Astrophysical Journal, 233:857-871, 1979 November 1

(C) 1979. The American Astronomical Society. All rights reserved. Printed in U.S.A.

\title{
THE EXCITATION OF DENSITY WAVES AT THE LINDBLAD AND COROTATION RESONANCES BY AN EXTERNAL POTENTIAL ${ }^{1}$
}

\author{
Peter Goldreich ${ }^{2}$ and SCOTt TRemaine ${ }^{3}$ \\ Received 1978 September 22; accepted 1979 May 2
}

\begin{abstract}
We calculate the linear response of a differentially rotating two-dimensional gas disk to a rigidly rotating external potential. The main assumptions are that the sound speed is much smaller than the orbital velocity and that the external potential varies on the scale of the disk radius. We investigate disks both with and without self-gravity.

The external potential exerts torques on the disk only at the Lindblad and corotation resonances. The torque is positive at the outer Lindblad resonance and negative at the inner Lindblad resonance; at corotation the torque has the sign of the radial gradient of vorticity per unit surface density. The torques are of the same order of magnitude at both types of resonance and are independent of the sound speed in the disk.

The external potential also excites density waves in the vicinity of the Lindblad and corotation resonances. The long trailing wave is excited at a Lindblad resonance. It transports away from the resonance all of the angular momentum which is deposited there by the external torque. Short trailing waves are excited at the corotation resonance. The amplitudes of the excited waves are the same on both sides of the resonance and are small unless the disk is almost gravitationally unstable. No net angular momentum is transported away from the corotation region by the waves. Thus the angular momentum deposited there by the external torque accumulates in the gas.

We briefly discuss the behavior of particle disks and prove that the external torques on particle disks are identical to those on gas disks.
\end{abstract}

Subject headings: galaxies: structure - hydrodynamics — stars: stellar dynamics

\section{INTRODUCTION}

Toomre (1969) and Kalnajs (1972) have shown that a tightly wound free spiral density wave cannot survive for longer than $10^{9} \mathrm{yr}$ in a typical spiral galaxy. Fresh waves must be continually created to maintain the spiral pattern. These waves might be excited by an external potential, perhaps due to a central bar or, in rare cases, to a satellite galaxy. Alternately, a galaxy may contain a density wave amplifier and a feedback mechanism for converting trailing into leading waves. This paper is mostly concerned with the former possibility.

We consider gaseous disks, although the mass fraction of gas in spiral galaxies is small. This approach is often fruitful because the hydrodynamic behavior and stellar dynamic behavior of galaxies are very similar. We discuss the applicability of our results to stellar systems in $\S \mathrm{V}$.

Much of the paper is devoted to justifying the validity of the approximations we make in deriving the basic equations and in solving Poisson's equation. These parts may be omitted without affecting the continuity of the paper. The main results are summarized in the Abstract, and a key to the principal equations is provided in $\S$ VI. Otherwise the plan of the paper is as follows. The basic equations which govern tightly wound density waves at the Lindblad resonances are described in $\S$ III. In $\S$ IV the driving of short trailing waves at the corotation resonance is calculated. A comparison of our results with those of other workers is presented in $\S \mathrm{V}$. Concluding remarks are contained in $\S$ VI.

\section{BASIC EQUATIONS}

We analyze a two-dimensional gas disk and adopt cylindrical coordinates $r, \theta, z$ and associated unit vectors $\hat{\boldsymbol{e}}_{r}, \hat{\boldsymbol{e}}_{\theta}, \hat{\boldsymbol{e}}_{z}$. The pressure $p$ acts only in the horizontal plane and is related to the surface mass density $\sigma$ by

$$
p=K \sigma^{\gamma} .
$$

We adopt this simple equation of state because our gas disk is partly intended to be an approximate analog to

${ }^{1}$ Contribution 3118 of the Division of Geological and Planetary Sciences, California Institute of Technology.

${ }^{2}$ California Institute of Technology.

${ }^{3}$ Institute of Astronomy, Cambridge, England; and Institute for Advanced Study, Princeton. 
stellar disks. A more general equation of state is considered by Kato and Inagaki (1978). The enthalpy $\eta$ and the sound speed $c$ satisfy

$$
(\gamma-1) \eta=c^{2}=\frac{d p}{d \sigma}
$$

The unperturbed disk rotates in the gravitational potential $\varphi_{0}(r) \cdot{ }^{4}$ Because the mass of the galactic halo may be comparable to the mass of the disk, $\varphi_{0}(r)$ and $\sigma_{0}(r)$ cannot be related by Poisson's equation and must be specified independently.

The unperturbed angular velocity is $\Omega(r)>0$, where

$$
\Omega^{2}(r)=\frac{1}{r} \frac{d}{d r}\left(\varphi_{0}+\eta_{0}\right) .
$$

The Oort parameter $B(r)$ and the epicyclic frequency $\kappa(r)$ are defined by

$$
B(r)=\Omega(r)+\frac{r}{2} \frac{d \Omega}{d r}
$$

and

The vorticity is $2 B(r)$.

$$
\kappa^{2}(r)=4 B(r) \Omega(r) .
$$

Consider the response of the disk to an external perturbation potential $\varphi_{1}(r, \theta, t)$. The surface density perturbation $\sigma_{1}$ is the source for an additional potential perturbation $\varphi_{1}{ }^{D}$. The linear perturbation equations read:

$$
\begin{aligned}
\frac{\partial \boldsymbol{v}_{1}}{\partial t}+\left(\boldsymbol{v}_{0} \cdot \boldsymbol{\nabla}\right) \boldsymbol{v}_{1}+\left(\boldsymbol{v}_{1} \cdot \boldsymbol{\nabla}\right) \boldsymbol{v}_{0} & =-\boldsymbol{\nabla}\left(\varphi_{1}+{\varphi_{1}}^{D}+\eta_{1}\right) \\
\frac{\partial \sigma_{1}}{\partial t}+\boldsymbol{\nabla} \cdot\left(\sigma_{0} \boldsymbol{v}_{1}\right)+\boldsymbol{\nabla} \cdot\left(\sigma_{1} \boldsymbol{v}_{0}\right) & =0 \\
\eta_{1} & =c_{0}^{2}\left(\sigma_{1} / \sigma_{0}\right) \\
\nabla^{2}{\varphi_{1}}^{D} & =4 \pi G \sigma_{1} \delta(z)
\end{aligned}
$$

where $v_{0}=r \Omega(r) \hat{e}_{\theta}$ and $\delta(z)$ is the Dirac delta function.

Without loss of generality, we write each perturbation variable $X$ in the form

$$
X=X(r) \exp i(m \theta-\omega t),
$$

where $m$ is a positive integer. In general, $X(r)$ is complex. However, the phase of the potential due to a bar or a satellite is independent of $r$. We set this phase equal to zero and take $\varphi_{1}(r)$ as real.

In component form, the momentum equation (5) becomes

$$
\begin{aligned}
& i(m \Omega-\omega) u_{1}-2 \Omega v_{1}=-\frac{d}{d r}\left(\varphi_{1}+{\varphi_{1}}^{D}+\eta_{1}\right), \\
& 2 B u_{1}+i(m \Omega-\omega) v_{1}=-\frac{i m}{r}\left(\varphi_{1}+\varphi_{1}{ }^{D}+\eta_{1}\right),
\end{aligned}
$$

where $\boldsymbol{v}_{1}=u_{1} \hat{\boldsymbol{e}}_{r}+v_{1} \hat{\boldsymbol{e}}_{\theta}$. Solving equations (10) yields

$$
\begin{aligned}
& u_{1}=-\frac{i}{D}\left[(m \Omega-\omega) \frac{d}{d r}+\frac{2 m \Omega}{r}\right]\left(\varphi_{1}+\varphi_{1}{ }^{D}+\eta_{1}\right), \\
& v_{1}=\frac{1}{D}\left[2 B \frac{d}{d r}+\frac{m}{r}(m \Omega-\omega)\right]\left(\varphi_{1}+\varphi_{1}{ }^{D}+\eta_{1}\right),
\end{aligned}
$$

where

$$
D=\kappa^{2}-(m \Omega-\omega)^{2} .
$$

${ }^{4}$ The unperturbed and perturbed variables carry subscripts 0 and 1 , respectively. 
We assume that the rotation curve is normal, that is, $D$ has a single maximum. Substituting equations (11) and (7) into the continuity equation (6), we obtain

$$
\left\{\frac{d^{2}}{d r^{2}}+\left[\frac{d}{d r} \ln \left(\frac{\sigma r}{D}\right)\right] \frac{d}{d r}+\frac{2 m \Omega}{r(m \Omega-\omega)}\left[\frac{d}{d r} \ln \left(\frac{\sigma \Omega}{D}\right)\right]-\frac{m^{2}}{r^{2}}\right\}\left(\varphi_{1}+\varphi_{1}{ }^{D}+\eta_{1}\right)=\frac{D \eta_{1}}{c^{2}},
$$

where the subscripts on $\sigma_{0}$ and $c_{0}$ have been dropped.

The response of the disk to $\varphi_{1}$ is completely described by equations (7), (8), and (13). Analytic solutions may be obtained for

$$
(c / \Omega r) \ll 1, \quad G \sigma / \Omega^{2} r \ll 1, \quad c^{2} / G \sigma r \ll 1,
$$

which we refer to as the tight-winding or Lin-Shu approximation (Lin and Shu 1968). In the solar neighborhood, $c \approx 30 \mathrm{~km} \mathrm{~s}^{-1}$ (appropriate for the $\mathrm{K}$ and $\mathrm{M}$ dwarfs which contain most of the disk mass), $\sigma \approx 75 M_{\odot} \mathrm{pc}^{-2}$. Thus $c / \Omega r \approx 0.1, G \sigma / \Omega^{2} r \approx 0.05, c^{2} / G \sigma r \approx 0.3$, so that the tight-winding approximation is not really appropriate. Nevertheless, analytic solutions obtained in this limit offer valuable insights into the behavior of density waves in more realistic model galaxies.

There are singularities in the coefficients of equation (13) where $D=0$ (Lindblad resonance, $r=r_{\mathrm{L}}$ ) and $m \Omega-\omega=0$ (corotation resonance, $r=r_{c}$ ). Away from the resonances, the general solution of the perturbation equations divides naturally into wave and non-wave parts. The individual waves satisfy the homogeneous equations $\left(\varphi_{1}=0\right)$ and are the free density waves of the Lin-Shu theory. The non-wave part is a particular solution of the inhomogeneous equations. Note that this division into homogeneous free-wave solutions and inhomogeneous non-wave solutions does not apply if the domain of the solutions includes the resonances. Then the relative magnitudes of the wave and non-wave parts are fixed by the behavior of $\varphi_{1}$ at the resonances and by the boundary conditions. We defer consideration of the resonances to later sections.

First consider the inhomogeneous non-wave solution. For this solution, the potential due to the surface density perturbation is negligible, so we set $\varphi_{1}{ }^{D}=0$. Then to lowest order in $c / \Omega r \ll 1$, we find

$$
\frac{\sigma_{1}}{\sigma}=\frac{\eta_{1}}{c^{2}}=\frac{1}{D}\left\{\frac{d^{2}}{d r^{2}}+\left[\frac{d}{d r} \ln \left(\frac{\sigma r}{D}\right)\right] \frac{d}{d r}+\frac{2 m \Omega}{r(m \Omega-\omega)}\left[\frac{d}{d r} \ln \left(\frac{\sigma \Omega}{D}\right)\right]-\frac{m^{2}}{r^{2}}\right\} \varphi_{1} .
$$

From Poisson's equation (8), we see that $O\left(\varphi_{1}{ }^{D}\right)=O\left(G \sigma_{1} r\right)=O\left(G \sigma \varphi_{1} / \Omega^{2} r\right)$ which justifies our neglect of $\varphi_{1}{ }^{D}$. A more careful examination of the magnitudes of the discarded terms in the vicinity of the resonances reveals that equation (15) is valid where $\left|\left(r-r_{\mathrm{L}}\right) / r_{\mathrm{L}}\right| \gg(c / \Omega r)^{1 / 2}$ and $\left|\left(r-r_{c}\right) / r_{c}\right| \gg(c / \Omega r)$.

Next consider the homogeneous free-wave solutions. The term $D \eta_{1} / c^{2}$ on the right-hand side of equation (13) can be balanced by terms on the left-hand side only if $O\left(d^{2} / d r^{2}\right) \gg r^{-2}$. Thus we look for solutions of the form

$$
\varphi_{1}^{D}(r)=\Phi(r) \exp \left[i \int^{r} k(s) d s\right]
$$

where $\Phi, k$ are real and $|k r| \gg 1$. For these rapidly oscillating solutions, Poisson's equation can be solved by a WKB approximation and yields

$$
\sigma_{1}=\frac{i \operatorname{sgn}(k)}{2 \pi G r^{1 / 2}} \frac{d}{d r}\left(r^{1 / 2} \varphi_{1}{ }^{D}\right)
$$

with fractional error $O\left(|k r|^{-2}\right.$ ) (Shu 1970). Substituting equations (16) and (17) into equation (13), setting $\varphi_{1}=0$, keeping the highest order terms in $k r$ and $\Omega r / c$, and separating real and imaginary terms, we obtain

and

$$
D+(k c)^{2}-2 \pi G \sigma|k|=0
$$

$$
\frac{d}{d r}\left[r \Phi^{2}\left(1-\frac{c^{2}|k|}{\pi G \sigma}\right)\right]=0
$$

Equation (18a) is the dispersion relation due to Lin and Shu (1968). Its solutions are

$$
|k|=\frac{\pi G \sigma}{c^{2}}+\epsilon\left[\left(\frac{\pi G \sigma}{c^{2}}\right)^{2}-\frac{D}{c^{2}}\right]^{1 / 2},
$$

where $\epsilon=+1$ for "short waves" and -1 for "long waves." The waves have a spiral form, and both leading $(k<0)$ and trailing $(k>0)$ waves are permitted. The group velocity $c_{g}$ is given by (Toomre 1969)

$$
c_{g} \equiv \frac{d \omega}{d k}=\operatorname{sgn} k\left[\frac{\pi G \sigma-|k| c^{2}}{m \Omega-\omega}\right] .
$$


For axisymmetric perturbations, $m=0$, equation (18a) reduces to

$$
\omega^{2}=\kappa^{2}-2 \pi G \sigma|k|+c^{2} k^{2} .
$$

Stable disks have $\omega^{2}>0$ for all $k$, which requires (Toomre 1964)

$$
Q=\frac{\kappa c}{\pi G \sigma}>1 \text {. }
$$

In the solar neighborhood $Q$ is probably between 1.2 and 2.0 (Toomre 1974). We take $Q$ to be of order unity in this investigation. The tight-winding approximation reduces to $O(c / \Omega r) \ll 1, O(Q)=1$.

Spiral density waves transport angular momentum by gravitational torques and by advective transport (LyndenBell and Kalnajs 1972). The angular momentum flux across a cylinder of radius $r$ due to gravitational torques is

$$
F_{G}=\frac{1}{4 \pi G} \int_{0}^{2 \pi} d \theta \int_{-\infty}^{\infty} d z r \operatorname{Re}\left[\frac{\partial \varphi_{1}{ }^{D}}{\partial \theta}\right] \operatorname{Re}\left[\frac{\partial \varphi_{1}{ }^{D}}{\partial r}\right] \text {. }
$$

The WKB solutions of Poisson's equation (8) for $z \neq 0$ show that

Thus

$$
\operatorname{Re}\left[\varphi_{1}{ }^{D}(r, \theta, z, t)\right]=\Phi(r) \exp (-|k(r) z|) \cos \left(\int^{r} k(s) d s+m \theta-\omega t\right) .
$$

$$
F_{G}=\operatorname{sgn}(k) \frac{m r \Phi^{2}(r)}{4 G}
$$

The flux due to advective transport is

$$
F_{A}=r^{2} \sigma \int_{0}^{2 \pi} d \theta \operatorname{Re}(u) \operatorname{Re}(v) .
$$

Evaluating $u$ and $v$ from equation (11) and setting $\varphi_{1}=0$ yields

$$
F_{A}=\frac{\pi m r \sigma}{D}\left[\operatorname{Im}\left(\varphi_{1}{ }^{D}+\eta_{1}\right) \operatorname{Re} \frac{d}{d r}\left(\varphi_{1}+\varphi_{1}{ }^{D}+\eta_{1}\right)-\operatorname{Re}\left(\varphi_{1}+\varphi_{1}{ }^{D}+\eta_{1}\right) \operatorname{Im} \frac{d}{d r}\left(\varphi_{1}{ }^{D}+\eta_{1}\right)\right],
$$

where $\omega$ is taken to be real. From equations (7), (16), and (17), we find

$$
{\varphi_{1}}^{D}+\eta_{1}=\left(1-\frac{c^{2}|k|}{2 \pi G \sigma}\right) \Phi(r) \exp \left[i \int^{r} k(s) d s\right]
$$

to lowest order in $(k r)^{-1}$. Thus for free waves $\left(\varphi_{1}=0\right)$

$$
F_{A}=-\frac{\pi m r \sigma k}{D}\left(1-\frac{c^{2}|k|}{2 \pi G \sigma}\right)^{2} \Phi^{2} .
$$

The total angular momentum flux is obtained from equations (25) and (29) with the aid of the dispersion relation (eq. [18a]) and reads

$$
F=F_{G}+F_{A}=-\operatorname{sgn}(k) \frac{m r \Phi^{2}}{4 G}\left(1-\frac{c^{2}|k|}{\pi G \sigma}\right) .
$$

Equation (18b) shows that the waves' angular momentum flux is conserved.

The general solution of the perturbation equations is a linear combination of the inhomogeneous nonwave solution and the homogeneous wave solutions. There is no angular momentum flux associated with the nonwave solution because, for it, $\sigma_{1}$ and $\varphi_{1}$ are in phase. The angular momentum flux due to cross-terms between different waves and between wave and nonwave solutions vanishes when averaged over radius.

Our conclusion is that, in the tight-winding limit, there is no transfer of angular momentum between the disk and the perturbing potential except at resonances. This conclusion depends upon the assumption that the external potential varies slowly, $\left|d \varphi_{1} / d r\right| \ll\left|k \varphi_{1}\right|$.

\section{LINDBLAD RESONANCES}

To solve equation (13) in the vicinity of a Lindblad resonance, we change the independent variable from $r$ to

$$
x \equiv\left(r-r_{\mathrm{L}}\right) / r_{\mathrm{L}}
$$


and replace $D$ by $\mathscr{D} x$, where

For $|x| \ll 1$, equation (13) simplifies to

$$
\mathscr{D} \equiv[r(d D / d r)]_{r_{\mathrm{L}}}
$$

$$
\left\{\frac{d^{2}}{d x^{2}}-\frac{1}{x} \frac{d}{d x}-\left[\frac{2 m \Omega}{(m \Omega-\omega)}\right]_{r_{\mathrm{L}}} \frac{1}{x}\right\}\left(\left(_{1}{ }^{D}+\eta_{1}\right)-\frac{\mathscr{D}}{c^{2}} r_{\mathrm{L}}{ }^{2} x \eta_{1}=\frac{\psi}{x},\right.
$$

with

$$
\Psi \equiv\left(r \frac{d \varphi_{1}}{d r}+\frac{2 m \Omega}{(m \Omega-\omega)} \varphi_{1}\right)_{r_{\mathrm{L}}},
$$

where we have used the fact that $O\left(d^{2} \varphi_{1} / d r^{2}\right)=O\left(r^{-1} d \varphi_{1} / d r\right)=O\left(r^{-2} \varphi_{1}\right)$. Next we drop the term on the lefthand side of equation (33) which is proportional to $[2 m \Omega /(m \Omega-\omega)]$ because $O\left[d\left(\varphi_{1}{ }^{D}+\eta_{1}\right) / d x\right] \gg O\left(\varphi_{1}{ }^{D}+\eta_{1}\right)$. In addition, we eliminate $\eta_{1}$ in favor of $\varphi_{1}{ }^{D}$ by using the WKB solution of Poisson's equation (eq. [17]) which simplifies to

$$
\frac{d \varphi_{1}^{D}}{d x}=-i \alpha \eta_{1}
$$

with

$$
\alpha \equiv\left(2 \pi G \sigma r / c^{2}\right)_{r_{L}} \operatorname{sgn}(k) .
$$

The validity of this procedure is established in the Appendix. The equation for $\varphi_{1}{ }^{D}$ now reads

where

$$
\left[\frac{d^{2}}{d x^{2}}-\left(i \alpha+\frac{1}{x}\right) \frac{d}{d x}+\frac{i \alpha}{x}-\beta x\right] \frac{d \varphi_{1}{ }^{D}}{d x}=-\frac{i \alpha}{x} \Psi,
$$

$$
\beta \equiv(r / c)_{r_{L}}^{2} \mathscr{D} .
$$

Note, $O\left(\alpha^{2}\right)=O(|\beta|)$ and $\operatorname{sgn} \beta= \pm 1$ for inner and outer Lindblad resonances. Equation (37) integrates once to yield

$$
\left(\frac{d^{2}}{d x^{2}}-i \alpha \frac{d}{d x}-\beta x\right) \varphi_{1}{ }^{D}=i \alpha \Psi
$$

This equation was derived and solved previously by Goldreich and Tremaine (1978a). A simpler method of solution is given here.

We introduce a new dependent variable

$$
w(x)=\varphi_{1}{ }^{D}(x) \exp (-i \alpha x / 2)
$$

and find

$$
\frac{d^{2} w}{d x^{2}}+\left(\frac{\alpha^{2}}{4}-\beta x\right) w=i \alpha \Psi \exp (-i \alpha x / 2)
$$

Since $O(|\beta|)=O\left(\alpha^{2}\right) \gg 1$, a WKB solution of equation (41) is valid for $|x| \ll 1$ and has the form ${ }^{5}$

where

$$
\begin{aligned}
w(x)= & \Psi\left[w_{S}(x) \int_{\mp \infty}^{x} d t w_{L}(t) \exp (-i \alpha t / 2)-w_{L}(x) \int_{\mp \infty}^{x} d t w_{S}(t) \exp (-i \alpha t / 2)\right] \\
& +M w_{S}(x)+N w_{L}(x)
\end{aligned}
$$

$$
w_{S}=\exp \left[\frac{1}{2} i \alpha x\left(1-\beta x / \alpha^{2}\right)\right], \quad w_{L}=\exp \left[-\frac{1}{2} i \alpha x\left(1-\beta x / \alpha^{2}\right)\right] .
$$

$M$ and $N$ are arbitrary constants. From the relation between $w$ and $\eta$, we see that $w_{S}$ and $w_{L}$ correspond to the short and the long waves. Furthermore, examination of the rate of phase variation of the integrands in equation (42) reveals that the external potential couples to the long wave near $x=0$ but does not couple to the short wave. In other words, the first integral in equation (42) is much smaller than the second for $\pm x \gg(|\alpha| /|\beta|)^{1 / 2}$ and is ignored from here on.

\footnotetext{
${ }^{5}$ In equation (42) and throughout the remainder of this section, the upper and lower signs apply to inner and outer Lindblad resonances.
} 
The correct expression for $\varphi_{1}{ }^{D}(x)$ is obtained by applying a radiation boundary condition to the general solution given in equation (42). That is, we demand that the solution contain only waves that propagate away from the Lindblad resonance. The direction of propagation of each type of density wave is given by the sign of its group velocity $c_{g}$ (eq. [20]). Inside corotation the group velocity is positive for long trailing and short leading waves and negatives for short trailing and long leading waves. Outside corotation the sign of the group velocity is reversed for each wave type. Long waves can exist only between the inner and outer Lindblad resonances whereas short waves are not restricted to this region (cf. eqs. [12] and [19]).

We are now prepared to choose the appropriate solution for $\varphi_{1}{ }^{D}(x)$. Consider first the solution for leading waves $(\alpha<0)$. We show that no solution exists. $N$ must vanish because $w_{L}(x)$ is trailing outside the Lindblad resonances (for $\pm x<0$ ). Then the contribution due to the second integral violates the radiation boundary condition between the Lindblad resonances (for $\pm x>0$ ). Next consider trailing waves $(\alpha>0)$. As before, $N=0$. The radiation boundary condition implies $M=0$. We have already discarded the first integral in equation (42). The only remaining term is the second integral which describes the excitation of the long trailing wave. The dominant contribution to this integral comes from a region of width $\Delta x \approx(|\alpha| /|\beta|)^{1 / 2}$ about the origin. This is where the long trailing wave varies most slowly and hence couples most strongly to the external potential.

The second term in brackets in equation (42), when multiplied by exp $(i \alpha x / 2)$, is a good approximation to $\varphi_{1}{ }^{D}(x)$. Thus

$$
\varphi_{1}^{D}(x) \approx-\Psi \exp \left(\frac{i \beta x^{2}}{2 \alpha}\right) \int_{\mp \infty}^{x} d t \exp \left(-\frac{i \beta t^{2}}{2 \alpha}\right) .
$$

An asymptotic expression for $\varphi_{1}{ }^{D}(x)$, valid for $|x| \gg(|\alpha| /|\beta|)^{1 / 2}$, is

$$
{\varphi_{1}}^{D}(x)=\left\{\frac{( \pm 1+\operatorname{sgn} x)}{2}\left(\frac{2 \pi|\alpha|}{|\beta|}\right)^{1 / 2} \exp \left[i\left(\frac{\beta x^{2}}{2 \alpha}+\frac{3 \pi}{4}\right)\right]-i\left(\frac{\alpha}{\beta}\right) \frac{1}{x}\right\} \Psi .
$$

The first term in the long trailing wave which exists on the corotation side of the Lindblad resonance. The nonwave part of the solution is given by the second term. With the aid of Poisson's equation (35) and the defining equations (36) and (38) for $\alpha$ and $\beta$, it is readily shown that the corresponding nonwave part of $\eta_{1}$ agrees with that given by equation (15) in the appropriate limit.

The long trailing wave excited at a Lindblad resonance has an angular momentum flux,

$$
F=-m \pi^{2}\left\{\left|\frac{\sigma}{r d D / d r}\right|\left[r \frac{d \phi_{1}}{d r}+\frac{2 m \Omega}{(m \Omega-\omega)} \varphi_{1}\right]^{2}\right\}_{r_{\mathrm{L}}}
$$

computed from equations (15), (19), (30), (32), and (34). Note that $F$ depends on $\sigma, \Omega$, and $\kappa$, but not on $c$ or $G$.

The behavior of a disk which is not self-gravitating may be investigated by setting $\varphi_{1}{ }^{D}=0$ in equation (33). In this case the long trailing waves propagate away from corotation. This changes the sign of the angular momentum flux but not its magnitude.

Further details concerning forced perturbations in the vicinity of a Lindblad resonance are given in the Appendix. It is demonstrated there that all the physical variables are well behaved and that the WKB solution of Poisson's equation is a valid approximation. We also prove that there is no accumulation of angular momentum in the vicinity of a Lindblad resonance. That is, the long trailing wave carries away all the angular momentum which the external torque deposits in the disk.

\section{COROTATION RESONANCE}

The corotation resonance occurs at the radius $r_{c}$ where $m \Omega\left(r_{c}\right)=\omega$. If the external potential is due to a nonaxisymmetric mass, such as a central bar, which rotates at constant angular speed $\Omega_{p}$, then $\omega=m \Omega_{p}$ and $\Omega\left(r_{c}\right)=\Omega_{p}$.

As before, we define a new variable

$$
x=\left(r-r_{c}\right) / r_{c} .
$$

Furthermore, we consider the response of the disk to a slowly increasing perturbation. Thus we take $\omega$ to have a small positive imaginary part and write

$$
\omega=m \Omega_{p}+i \epsilon,
$$

where $\epsilon>0$. The resonant term in equation (13) is proportional to

$$
(m \Omega-\omega)^{-1} \approx\left\{(m r d \Omega / d r)_{r_{c}}\left[x-\frac{i \epsilon}{(m r d \Omega / d r)_{r_{c}}}\right]\right\}^{-1} .
$$

Since $m>0$ by definition, and $d \Omega / d r<0$ for typical galaxies, the imaginary term is positive. In future, we drop all poles slightly below the real axis. 
Although our primary concern is with disks for which $O(Q)=1$, it is illuminating to consider first disks which are not self-gravitating, i.e., disks with $\varphi_{1}{ }^{D}=0$.

\section{a) Disks without Self-Gravity}

Density waves cannot propagate in the region between the inner and outer Lindblad resonances in disks without self-gravity (cf. eqs. [12] and [19]). Thus an external potential does not excite density waves at the corotation resonance in such disks. However, it is still worth investigating the nonwave forced perturbation in these disks for the insight it provides concerning the nature of forced disturbances in self-gravitating disks.

To reduce equation (13) to a tractable form in the neighborhood of corotation, we assume that $O\left(d^{2} \eta_{1} / d x^{2}\right) \gg$ $O\left(d \eta_{1} / d x\right) \gg O\left(\eta_{1}\right)$. Then keeping only the largest terms, we obtain

$$
\frac{d^{2} \eta_{1}}{d x^{2}}+\left(\frac{p}{x}-q^{2}\right) \eta_{1}=-\frac{p}{x} \varphi_{1}\left(r_{c}\right)
$$

where

$$
p=\left[\frac{2 \Omega}{d \Omega / d r} \frac{d}{d r} \ln \left(\frac{\sigma \Omega}{D}\right)\right]_{r_{c}}=\left[\frac{2 \Omega}{d \Omega / d r} \frac{d}{d r} \ln \left(\frac{\sigma}{B}\right)\right]_{r_{c}}
$$

and

$$
q=(\kappa r / c)_{r_{c}}
$$

We simplify equation (50) further by assuming for the moment that $O\left(\eta_{1}\right) \ll O\left(\varphi_{1}\right)$ so that we can discard the term $p \eta_{1} / x$. The solution of the resulting equation is straightforward (Donner 1978):

$$
\eta_{1}=+\frac{p}{2 q} \varphi_{1}\left(r_{c}\right)\left[\exp (q x) \int_{x}^{\infty} \frac{d t}{(t+i \epsilon)} \exp (-q t)+\exp (-q x) \int_{-\infty}^{x} \frac{t}{(t+i \epsilon)} \exp (q t)\right],
$$

where we have imposed the boundary condition $\eta_{1} \rightarrow 0$ as $|x| \rightarrow \infty$. Here $0<\epsilon \ll 1$. The asymptotic solution for $\eta_{1}$ follows immediately from the asymptotic behavior of exponential integrals (Abramowitz and Stegun 1964). We find

$$
\eta_{1}=\frac{p}{q^{2} x} \varphi_{1}\left(r_{c}\right)-\frac{i \pi p}{2 q} \varphi_{1}\left(r_{c}\right) \exp (-|q x|),
$$

for $q^{-1}=(c / \kappa r)_{r_{c}} \ll|x| \ll 1$. The first term matches onto the nonwave inhomogeneous solution for $\eta_{1}$ given by equation (15). The second term matches onto homogeneous solutions consistent with the dispersion relation (19).

The solution for $\eta_{1}$ has the property that $O\left(d^{2} \eta_{1} / d x^{2}\right) \gg O\left(d \eta_{1} / d x\right) \gg O\left(\eta_{1}\right)$, which validates the assumption made in deriving equation (50) from equation (13). To justify the neglect of the term $p \eta_{1} / x$ in equation (53), we note from equation (53) that $O\left(\eta_{1}\right)=q^{-1} O\left(\varphi_{1}\right) \ll O\left(\varphi_{1}\right)$.

Next we calculate the angular momentum flux near corotation. Since $\varphi_{1}{ }^{D}=0$, the entire flux is carried by advective transport. Substituting equation (53) into equation (27), we obtain

$$
F_{A}=-\frac{\pi^{2}}{4} m \operatorname{sgn}(x)\left[\frac{\varphi_{1}^{2}}{d \Omega / d r} \frac{d}{d r}\left(\frac{\sigma}{B}\right)\right]_{r_{c}} \exp (-|q x|),
$$

to lowest order in $(c / \Omega r)$. Note that $F_{A} \rightarrow 0$ as $|x| \rightarrow \infty$, so angular momentum is not transported to infinity. Curiously, there is a net flux of angular momentum into the resonance:

$$
F_{A}(-0)-F_{A}(+0)=\frac{\pi^{2} m}{2}\left[\frac{\varphi_{1}^{2}}{d \Omega / d r} \frac{d}{d r}\left(\frac{\sigma}{B}\right)\right]_{r_{c}} .
$$

The rate of accumulation of angular momentum is equal to the net torque exerted by the external potential in the neighborhood of corotation.

From equation (53) we see that, as $x \rightarrow 0, \eta_{1} \rightarrow-p \varphi_{1}\left(r_{c}\right)[i \pi /(2 q)+x \ln x]$. Thus the azimuthal velocity perturbation $v_{1} \propto \ln x$ for $|x| \ll q^{-1}$. This singularity is discussed further in $\S \S \operatorname{IV} b$ and V.

\section{b) Disks with Self-Gravity}

In self-gravitating disks, density waves can propagate between the inner and outer Lindblad resonances except in a forbidden region around corotation for which $\left|\Omega-\Omega_{p}\right|<\kappa\left(1-Q^{-2}\right)^{1 / 2} / m$ (cf. eq. [19]). The forbidden region is narrow if $0<Q-1 \ll 1$ and absent if $Q<1$ (unstable disks). Therefore, we expect the efficiency of density wave excitation to increase as $Q$ decreases. In this subsection we assume $O(|Q-1|) \ll 1$ so that for stable disks the forbidden zone occupies a small fraction of the region between the inner and outer Lindblad resonances. However, we consider both limits $1 \gg O(|Q-1|) \gg c / \Omega r$ and $O(|Q-1|) \ll c / \Omega r$. 
Once again, we are faced with reducing the equation governing forced disturbances (eq. [13]) to a simplified form valid near the corotation resonance. We assume that $O\left[d\left(\varphi_{1}{ }^{D}+\eta_{1}\right) / d x\right] \gg O\left[\left(\varphi_{1}{ }^{D}+\eta_{1}\right)\right]$. For $x \ll 1$, equation (13) then yields

$$
\left\{\frac{d^{2}}{d x^{2}}+\left[\frac{d}{d x} \ln \left(\frac{\sigma r}{D}\right)\right] \frac{d}{d x}+\frac{p}{x}\right\}\left(\varphi_{1}{ }^{D}+\eta_{1}\right)-\frac{D}{c^{2}} \eta_{1}=-\frac{p}{x} \varphi_{1}\left(r_{c}\right) .
$$

We assume for the moment that $O\left(\varphi_{1}{ }^{D}+\eta_{1}\right) \ll O\left(\varphi_{1}\right)$ so that we can neglect the term proportional to $p x^{-1}$ on the left-hand side of equation (57). The WKB solution of Poisson's equation (17) is written as

$$
\eta_{1}=\frac{i \operatorname{sgn}(k)}{2 k_{c} r^{1 / 2}} \frac{d}{d x}\left(r^{1 / 2} \varphi_{1}{ }^{D}\right)
$$

where

$$
k_{c}(x)=\pi G \sigma r_{c} / c^{2} .
$$

The validity of equation (58) is established in the Appendix. Equation (58) is now used to eliminate $\varphi_{1}{ }^{D}$ from equation (57) in favor of $\eta_{1}$. The result is

$$
\frac{d^{2} \eta_{1}}{d x^{2}}+\left[\frac{d}{d x} \ln \left(\frac{\sigma r}{D}\right)-2 i k_{c}\right] \frac{d \eta_{1}}{d x}+\left[-\frac{D}{c^{2}}-2 i \frac{d k_{c}}{d x}+\frac{i k_{c} r_{c}}{r}-2 i k_{c} \frac{d}{d x} \ln \left(\frac{\sigma r}{D}\right)\right] \eta_{1}=-\frac{p}{x} \varphi_{1}\left(r_{c}\right) .
$$

Next introduce a new dependent variable

Then set

$$
y(x)=(\sigma r / D)^{1 / 2} \exp \left[-i k_{c} \operatorname{sgn}(k) x\right] \eta_{1}(x) .
$$

$$
D \equiv \kappa^{2}-\left[m\left(\Omega-\Omega_{p}\right)\right]^{2} \approx \kappa^{2}\left(1-x^{2} / L^{2}\right)
$$

where

$$
\frac{1}{L}=\left|\frac{m r}{\kappa} \frac{d \Omega}{d r}\right|_{r_{c}} .
$$

Note, $O(L)=1$. We retain the $x$ dependence of $D$ near corotation so that our final equation can describe the transition from evanescent to propagating disturbances which occurs at the boundary of the forbidden region. With these changes, equation (60) becomes

$$
\frac{d^{2} y}{d x^{2}}+k_{c}^{2}\left(1-Q^{2}+\frac{Q^{2} x^{2}}{L^{2}}+\frac{2 i}{k_{c}} \frac{d}{d x} \ln Q\right) y=-\frac{p}{x}\left(\frac{\sigma r}{D} \varphi_{1}\right)_{r_{c}} \exp \left[-i k_{c} \operatorname{sgn}(k) x\right] .
$$

In deriving equation (64) we have discarded terms of order unity in the coefficient of $y$. Henceforth we take $Q$ to be constant and evaluate $k_{c}$ at $x=0$. Finally, we define

$$
\begin{aligned}
v & =\left(2 k_{c} Q / L\right)^{1 / 2} x, \\
b & =k_{c} L\left(Q^{2}-1\right) / 2 Q, \\
K & =\operatorname{sgn}(k)\left(k_{c} L / 2 Q\right)^{1 / 2}, \\
S & =-p\left(\frac{L \sigma c}{2 \kappa^{3}}\right)_{r_{c}}^{1 / 2} \varphi_{1}\left(r_{c}\right),
\end{aligned}
$$

and rewrite equation (64) as

$$
\frac{d^{2} y}{d v^{2}}+\left(-b+\frac{v^{2}}{4}\right) y=\frac{S}{v} \exp (-i K v)
$$

The stellar dynamical analog of the homogeneous part of this equation was originally derived by Mark (1976). Lin and Lau (1975) derived equation (69) and studies its solution in some special cases.

It turns out to be most convenient to solve equation (60) in the interval $-\infty<v<\infty$, although it is only a valid approximation to the dynamics for $|v| \ll\left(2 k_{c} Q \mid L\right)^{1 / 2}(|x| \ll 1)$. This procedure introduces a minor problem which is easily overcome by a slight modification of the inhomogeneous term. The difficulty is due to the expression adopted for $D$ (eq. [62]) which vanishes at $|v|=\left(2 k_{c} Q L\right)^{1 / 2}(|x|=L)$. These locations have some of the properties of Lindblad resonances. In particular, the inhomogeneous term generates small propagating disturbances there. Since we are interested in isolating the effects of driving at the corotation resonance, we eliminate 
this unwanted (and invalid) source of waves by multiplying the inhomogeneous term by $f(v)$, where $f(v)=1$ near $v=0$ and declines monotonically to $f(v)=0$ for $|v|<\left(2 k_{c} L Q\right)^{1 / 2}$. We show that the driving near corotation occurs in the interval $O(K v)=O\left(k_{c} x\right)=1$. Thus as long as $f(v)=1$ in this interval, it does not affect ! the excitation of the waves.

The homogeneous solutions of equation (69) are parabolic cylinder functions (Abramowitz and Stegun 1964). It is most convenient to take $E(b, v)$ and $E^{*}(b,-v)$ as the independent homogeneous solutions. Relevant properties of these solutions are

and

$$
\begin{gathered}
E^{*}(b, v)=+i \exp (\pi b) E^{*}(b,-v)-i[1+\exp (2 \pi b)]^{1 / 2} E(b,-v), \\
E(b, v) \underset{v \gg|b|^{1 / 2}}{\longrightarrow}\left(\frac{2}{v}\right)^{1 / 2} \exp \left\{i\left[\frac{v^{2}}{4}-b \ln v+\frac{\delta}{2}+\frac{\pi}{4}\right]\right\} \\
\delta=\arg \left[\Gamma\left(\frac{1}{2}+i b\right)\right]
\end{gathered}
$$

$$
W\left[E(b, v), E^{*}(b,-v)\right]=2 \exp (\pi b),
$$

where $W$ denotes the Wronskian. The general solution of equation (69) may be written as

$$
\begin{aligned}
y(v)=-\frac{S}{2} \exp (-\pi b)\{ & E(b, v)\left[\int_{-\infty}^{v} d t \frac{E^{*}(b,-t)}{(t+i \epsilon)} f(t) \exp (-i K t)+M\right] \\
& \left.+E^{*}(b,-v)\left[\int_{v}^{\infty} d t \frac{E(b, t)}{(t+i \epsilon)} f(t) \exp (-i K t)+N\right]\right\},
\end{aligned}
$$

where $M$ and $N$ are arbitrary constants.

We impose outward radiation boundary conditions on $\eta_{1}[x(v)]$ for $|v|>2 b^{1 / 2}$. Long leading waves and short trailing waves propagate away from the boundary of the forbidden zone around corotation $\left(|v|=2 b^{1 / 2}\right)$ toward the inner and outer Lindblad resonances. It is easy to verify that, for both leading and trailing disturbances, this implies $M=N=0$.

To evaluate the integrals in equation (69), we make use of the fact that $K \gg 1$. We define

$$
\begin{aligned}
& I_{1}(v) \equiv \int_{-\infty}^{v} d t \frac{E^{*}(b,-t)}{(t+i \epsilon)} f(t) \exp (-i K t), \\
& I_{2}(v) \equiv \int_{v}^{\infty} d t \frac{E(b, t)}{(t+i \epsilon)} f(t) \exp (-i K t) .
\end{aligned}
$$

Consider first

$$
I_{1}(\infty)=-i \pi E^{*}(b, 0)+\frac{1}{2} \int_{-\infty}^{\infty} \frac{d t}{t} f(t)\left\{\cos K t\left[E^{*}(b,-t)-E^{*}(b, t)\right]-i \sin K t\left[E^{*}(b,-t)+E^{*}(b, t)\right]\right\} .
$$

In the limit $|K| \rightarrow \infty$, the integral containing $\cos K t$ vanishes and the integral containing sin $K t$ is evaluated by setting $\sin K t / t=\pi \delta(t) \operatorname{sgn}(k)$. Thus

Similarly,

$$
I_{1}(\infty)=-i \pi[1+\operatorname{sgn}(k)] E^{*}(b, 0)
$$

$$
I_{2}(-\infty)=-i \pi[1+\operatorname{sgn}(k)] E(b, 0)
$$

The amplitudes of the waves which propagate toward $+\infty$ and $-\infty$ are proportional to $I_{1}(\infty)$ and $I_{2}(-\infty)$, respectively. Since $I_{1}(\infty)$ and $I_{2}(-\infty)$ vanish for $\operatorname{sgn}(k)=-1$, leading waves are not excited at corotation. This conclusion was first reached by Feldman and Lin (1973). Since $\left|I_{1}(\infty)\right|=\left|I_{2}(-\infty)\right|$, the trailing waves are excited with the same strength on both sides of corotation.

The nonwave part of $y(v)$ is proportional to

$$
J_{1}(v) \equiv E(b, v)\left[I_{1}(v)-I_{1}(\infty)\right]+E^{*}(b,-v) I_{2}(v) \quad \text { for } v \gg K^{-1},
$$

and to

$$
J_{2}(v)=E(b, v) I_{1}(v)+E^{*}(b,-v)\left[I_{2}(v)-I_{2}(-\infty)\right] \text { for } v \ll-K^{-1} .
$$

To evaluate $J_{1}(v)$ for $v \gg K^{-1}$, we form

$$
\frac{d J_{1}}{d v}(v) \approx\left[\frac{d E^{*}}{d v}(b,-v) E(b, v)-\frac{d E}{d v}(b, v) E^{*}(b,-v)\right] f(v) \int_{v}^{\infty} \frac{d t}{t} \exp (-i K t),
$$


where we have taken advantage of the fact that $E(b, v), E^{*}(b, v)$, and $f(v)$ are all slowly varying compared to $\exp (-i K v)$ and can be taken out of the integrals. The angular bracket contains the Wronskian of $E(b, v)$, $E^{*}(b,-v)$ (cf. eq. [73]), and the remaining integral is well approximated by $-i(K v)^{-1} \exp (-i K v)$. Once $\therefore d J_{1}(v) / d v$ is known, $J_{1}(v)$ is obtained by integration using $J_{1}(\infty)=0$. In this manner we obtain

$$
J_{1}(v)=2 f(v)\left(K^{2} v\right)^{-1} \exp [\pi b-i K v] \text { for } v \gg K^{-1} .
$$

Similarly,

$$
J_{2}(v)=2 f(v)\left(K^{2} v\right)^{-1} \exp [\pi b-i K v] \text { for } v \ll-K^{-1} .
$$

The nonwave part of $\eta_{1}(x)$ near corotation is determined by equations (61), (65)-(68), (74), (83), and (84). It reads

$$
\left.\eta_{1}(x)\right|_{n-w}=\left\{\frac{2 \Omega}{d \Omega / d r}\left[\frac{d}{d r} \ln \left(\frac{\sigma \Omega}{D}\right)\right]\left(\frac{c}{K r}\right)^{2} \varphi_{1}\right\}_{r_{c}} \frac{1}{x},
$$

for $k_{c}{ }^{-1} \ll|x| \ll 1$. In deriving equation (85), we have set $k_{c} \approx(\kappa r / c)$, consistent with our assumption $|(Q-1) / Q| \ll 1$. The above expression agrees with the more general nonwave solution for $\eta_{1}$ (cf. eq. [15]) near corotation.

The contributions to $\eta_{1}[x(v)]$ from the short trailing waves excited at corotation are given by equations (61), (74), (78), and (79). They are

$$
\left.\eta_{1}[x(v)]\right|_{w}=i \pi S \exp (-\pi b) E^{*}(b, 0) E(b, v) \exp (i K v) \quad \text { for } v \gg K^{-1}
$$

and

$$
\left.\eta_{1}[x(v)]\right|_{w}=i \pi S \exp (-\pi b) E(b, 0) E^{*}(b,-v) \exp (i K v) \text { for } v \ll-K^{-1} .
$$

We are now in a position to justify the assumptions made in the derivation of equation (60). From equations (61), (74), (85), and (86), we see that $O\left(d^{2} \eta_{1} / d x^{2}\right) \gg O\left(d \eta_{1} / d x\right) \gg O\left(\eta_{1}\right)$. Equation (58) then shows that $O\left(\varphi_{1}{ }^{D}\right)=$ $O\left(\eta_{1}\right)$. To estimate $\eta_{1}$ we note that for $b>0$ (stable disks), $O[E(b, v)] \leq \exp (-\pi b / 2)$. Hence $O\left(\eta_{1}\right)=O\left(\varphi_{1}{ }^{D}\right) \leq$ $O(S)=O\left[(c / \Omega r)^{1 / 2} \varphi_{1}\right] \ll O\left(\varphi_{1}\right)$, which justifies our neglect of $\left(\eta_{1}+\varphi_{1}{ }^{D}\right) / x$ with respect to $\varphi_{1} / x$. For $b<0$ (unstable disks), $O[E(b, 0)]=1$ and $O\left(\eta_{1}\right)=O\left(\phi_{1}{ }^{D}\right)=O\left[(c / \Omega r)^{1 / 2} \exp (|\pi b|) \varphi_{1}\right]$ for $x \approx 0$. Thus $O\left(\eta_{1}+\varphi_{1}{ }^{D}\right) \ll$ $O\left(\phi_{1}\right)$ if $|\pi b| \ll \ln (\Omega r / c)$. For larger values of $|b|$, the forced perturbations are so large that our approximations fail. Unstable disks probably do nor merit special study since they are unlikely to exist in nature.

The short trailing waves excited at corotation carry angular momentum toward $x= \pm \infty$. We evaluate the angular momentum flux near corotation from equations (19), (30), and (62). Well outside the forbidden region $\left[|x| \gg\left(Q^{2}-1\right)^{1 / 2} L / Q\right]$,

$$
F \approx \frac{m r_{c} \Phi^{2}}{4 G} \frac{Q}{L}|x|
$$

$F$ is positive on both sides of corotation because the angular momentum density is positive for $x>0$ and negative for $x<0$. Near corotation, the wave part of the perturbation satisfies $\Phi \equiv\left|\varphi_{1}^{D}\right|=2\left|\eta_{1}\right|$. Using this relation together with equations (65)-(68), (70), (71), and (87), we obtain

$$
F=\frac{\pi^{4} \sigma Q^{2}}{\kappa^{2}}\left\{\left|\frac{m c}{r^{2} d \Omega / d r}\right|^{1 / 2}\left[\frac{2 \Omega \varphi_{1}}{(d \Omega / d r)} \frac{d \ln }{d r}\left(\frac{\sigma}{B}\right)\right]^{2}\right\}_{r_{c}} \frac{\exp (-3 \pi b / 2)}{\left|\Gamma\left(\frac{3}{4}+i b / 2\right)\right|^{2}}
$$

where we have explicitly evaluated $|E(b, 0)|^{2}$. For large $|b|,\left|\Gamma\left(\frac{3}{4}+i b / 2\right)\right|^{2} \rightarrow \pi|2 b|^{1 / 2} \exp (-\pi|b| / 2)$. Thus $F \propto$ $\exp (-\pi b)$ for $b>0$, and $F \propto \exp (2 \pi|b|)$ for $b<0$. From the definition of $b$ (cf. eq. [66]), we see that for stable disks, $F$ is negligible unless $O(Q-1)=O(c / \Omega r) \ll 1$. Even if $Q=1, F$ for the waves excited at corotation is smaller by $O\left[(c / \Omega r)^{1 / 2}\right] \ll 1$ than $F$ for the waves excited at the Lindblad resonances.

The external potential exerts a torque on the disk

$$
T=-\int_{-\infty}^{\infty} r d r \int_{0}^{2 \pi} d \theta \sigma_{1}(r, \theta)\left(r \times \nabla \varphi_{1}\right) \cdot \hat{\boldsymbol{e}}_{z} .
$$

Since $\varphi_{1}(r, \theta, t)=\varphi_{1}(r) \exp [i(m \theta-\omega t)]$ with $\varphi_{1}(r)$ real,

$$
T_{c} \approx-m \pi\left(r^{2} \sigma \varphi_{1} / c^{2}\right)_{r_{c}} \int_{-\infty}^{\infty} d x g(x) \operatorname{Im}\left(\eta_{1}\right),
$$

where we have evaluated $\varphi_{1}(r)$ at $r=r_{c}$ and replaced $\sigma_{1}(r)$ by $\eta_{1}(r)$ using equation (7). The auxiliary function $g(x)$ has been inserted in the integrand for reasons explained below. Near corotation $\eta_{1}[v(x)]=\exp (i K v) y(v)$, where $y(v)$ is given by equation (74). Since $\eta_{1}$ oscillates, the torque density oscillates as well. Nevertheless, the net torque 
in the corotation region is well defined by equation (90) if we take $g(0) \approx 1$ for $|x| \lesssim k_{c}{ }^{-1}$ and let $g(x) \rightarrow 0$ smoothly for $|x| \gg k_{c}^{-1}$. The effect of $g(x)$ is to slowly damp the oscillations in torque density and thus to produce a finite, well-defined, expression for $T_{c}$ which is essentially independent of $g(x)$.

The most economical method of computing $T_{c}$ is to use equation (69) as follows. Define

$$
J \equiv \int_{-\infty}^{\infty} d v \exp \left[i K v-(\Delta v)^{2}\right]\left[\frac{d^{2} y}{d v^{2}}+y\left(-\frac{b+v^{2}}{4}\right)\right]=-i \pi S
$$

where $g[v(x)]=\exp \left[-(\Delta v)^{2}\right]$ with $K^{1 / 2} \ll \Delta \ll K$. For this choice of $\Delta$, the $v^{2} y / 4$ term in the integrand may be neglected with respect to the $d^{2} y / d v^{2}$ term. Integration by parts then yields

$$
J \simeq-\left(K^{2}+b\right) \int_{-\infty}^{\infty} d v \exp \left[i K v-(\Delta v)^{2}\right] y(v)=-i \pi S,
$$

where the discarded terms in the integrand are $O\left(\Delta K^{-1}\right) \ll 1$ smaller than those retained. From equations $(90)$ and (92) we obtain

$$
T_{c}=\frac{m \pi^{2}}{2}\left[\frac{\varphi_{1}^{2}}{d \Omega / d r} \frac{d}{d r}\left(\frac{\sigma}{B}\right)\right]_{r_{c}}
$$

As advertised, this result is independent of $g[v(x)]$ or, more precisely, of the value of $\Delta$. Comparison of equations (56) and (93) reveals that the net torque at corotation is independent of whether or not the disk is self-gravitating. Self-gravitating disks share another property with non-self-gravitating disks. From equation (69) it follows that $\eta_{1}[v(x)] \propto v \ln v$ as $v \rightarrow 0$. This implies that the azimuthal velocity perturbation varies as $\ln x$ as $x \rightarrow 0$ (cf. eq. [12]).

\section{DISCUSSION}

The behavior of a disk near the Lindblad and corotation resonances has been treated previously in several papers. Below we describe how the results of these earlier papers compare with those presented here. We discuss results obtained for gas disks first.

The excitation of the long trailing wave at the Lindblad resonances by an external potential was calculated by Goldreich and Tremaine $(1978 a, b)$ for self-gravitating disks. Donner (1978) derived the wave driving at the Lindblad resonances for disks without self-gravity. In appropriate limits, the results of these earlier works are compatible with those reported in the current paper.

The study of the corotation resonance most closely comparable to ours is by Feldman and Lin (1973). Their equation (6.13) is analogous to our equation (60) in the limit $|x| \ll L\left(Q^{2}-1\right)^{1 / 2} / Q$. Feldman and Lin were the first to show that an external potential excites the short trailing wave at corotation. However, they did not compute the angular momentum flux of the driven waves. Lin and Lau (1975) concentrated on the special case $Q=1$ and on cylindrical rather than disk geometry. Subsequently, Lau (1977) derived the wave driving at corotation in cylindrical geometry. He established that the amplitudes of the excited waves are the same on both sides of corotation.

Donner (1978) solved the initial value problem for the forcing of disks without self-gravity. He included the effects of bulk viscosity. His paper contains the first derivation of equation (53) describing the response near corotation.

Kato and Inagaki (1978) discuss angular momentum transport near the corotation resonance of a disk without self-gravity. They use a more general equation of state than we do and consider nonadiabatic perturbations. However, their work is restricted to disks with very small differential rotation and is not directly comparable to ours.

The most interesting comparison of our work is with the stellar dynamical calculations of Lynden-Bell and Kalnajs (1972, hereafter LBK). These authors determined the rate $\dot{H}$ at which a disk of stars gains angular momentum under the influence of a potential of the form $\varphi_{1}(r) \exp [i(m \theta-\omega t)]$. We assume that the disk is not selfgravitating, so that $\varphi_{1}$ is the external potential. According to their equation (30)

$$
\dot{H}=-\frac{1}{8 \pi} \sum_{l, m} \int_{0}^{\infty} \int_{0}^{\infty} m\left(l \frac{\partial F}{\partial J_{1}}+m \frac{\partial F}{\partial J_{2}}\right)\left|\varphi_{l m}\right|^{2} \delta\left(l \Omega_{1}+m \Omega_{2}-\omega\right) d J_{1} d J_{2} .
$$

Here $J_{1}$ is the radial action, $J_{2}$ is the azimuthal action or orbital angular momentum, and $F\left(J_{1}, J_{2}\right)$ is the distribution function. $F$ gives the mass density per unit volume in the four-dimensional phase space. In the limit 
$J_{1} / \kappa r^{2} \ll 1$ (corresponding to the epicyclic approximation or to our limit $\left.c / \Omega r \ll 1\right), \Omega_{1}=\kappa$ and $\Omega_{2}=\Omega(r)$. The Fourier components of the potential $\varphi_{l m}$ are related to our $\varphi_{1}(r)$ by

$$
\begin{array}{rlrl}
\left|\varphi_{l m}\right|^{2} & =16 \pi^{4}{\varphi_{1}}^{2}, & l=0, \\
& =\frac{8 \pi^{4} J_{1}}{\kappa r^{2}}\left(\frac{r d \varphi_{1}}{d r}-\frac{2 m \Omega \varphi_{1}}{l \kappa}\right)^{2}, & & |l|=1, \\
& \lesssim 0\left[{\varphi_{1}}^{2}\left(\frac{J_{1}}{\kappa r^{2}}\right)^{2}\right], & & |l|>1 .
\end{array}
$$

To derive equation (95) we used equation (18) from LBK and assumed $O\left(d \varphi_{1} / d r\right)=O\left(\varphi_{1} / r\right)$. Note that when equation (95) is substituted into equation (94), $\varphi_{1}(r), \kappa(r), \Omega(r)$, and $r$ are considered functions of $J_{2}$ through the relation $J_{2}=r^{2} \Omega(r)$. The argument of the delta function in equation (94) indicates that $l=0$ corresponds to the corotation resonance and $l= \pm 1$ correspond to the outer and inner Lindblad resonances, respectively.

For $l=0$, equations (94) and (95) yield

$$
\dot{H}=-\frac{\pi}{2} m^{2} \int_{0}^{\infty} d J_{2} \varphi_{1}{ }^{2} \delta(m \Omega-\omega) \frac{d}{d J_{2}}\left[4 \pi^{2} \int_{0}^{\infty} d J_{1} F\left(J_{1}, J_{2}\right)\right] .
$$

The quantity in the square brackets is the total mass in the interval $d J_{2}$ since the volume element in phase space is $d w_{1} d w_{2} d J_{1} d J_{2}$, where $w_{1}$ and $w_{2}$ are the angle variables. Since $J_{2}=r^{2} \Omega(r)$,

$$
4 \pi^{2} \int_{0}^{\infty} F d J_{1}=\frac{2 \pi r \sigma(r)}{d\left(r^{2} \Omega\right) / d r}=\frac{\pi \sigma}{B}
$$

Thus

$$
\dot{H}=-\frac{\pi^{2}}{2} m \int_{0}^{\infty} d r \varphi_{1}{ }^{2} \delta\left(\Omega-\Omega_{p}\right) \frac{d}{d r}\left(\frac{\sigma}{B}\right)=\frac{\pi^{2}}{2} m\left[\frac{\varphi_{1}{ }^{2}}{d \Omega / d r} \frac{d}{d r}\left(\frac{\sigma}{B}\right)\right]_{r_{c}}
$$

assuming $d \Omega / d r<0$. The final expression for $\dot{H}$ is identical to the result we obtained for the net external torque in the neighborhood of corotation (cf. eqs. [56] and [93]).

Next consider $|l|=1$. We drop the term proportional to $m \partial F / \partial J_{2}$ in equation (94) because its contribution to the integral is much smaller than that due to the term proportional to $l \partial F / \partial J_{1}$ ( $F$ is strongly peaked at small values of $J_{1}$ ). Integrating by parts and using equation (95), we obtain

$$
\dot{H}=\frac{\pi}{4} l m \int_{0}^{\infty} \frac{d J_{2}}{\kappa r^{2}}\left(r \frac{d \varphi_{1}}{d r}-\frac{2 m \Omega}{l \kappa} \varphi_{1}\right)^{2} \delta(l \kappa+m \Omega-\omega)\left[4 \pi^{2} \int_{0}^{\infty} d J_{1} F\left(J_{1}, J_{2}\right)\right] .
$$

With the aid of equation (97), $\dot{H}$ reduces to

$$
\dot{H}=\operatorname{lm} \pi^{2}\left\{\left|\frac{\sigma}{r d D / d r}\right|\left[r \frac{d \varphi_{1}}{d r}+\frac{2 \Omega}{\left(\Omega-\Omega_{p}\right)} \varphi_{1}\right]^{2}\right\}_{r_{\mathrm{L}}},
$$

where we have used $d D / d r=2 \kappa d(\kappa+\ln \Omega) / d r$. The above expression for $\dot{H}$ is equal to the external torque exerted on a gas disk in the neighborhood of the Lindblad resonance (eq. [10]). However, in the gas disk all of the angular momentum deposited by the torque is transported away by a long trailing wave (see Appendix), while in the stellar disk without self-gravity the angular momentum is absorbed by the stars near the resonance and no density wave is excited.

The case of a self-gravitating stellar disk is more complicated. The dispersion relation for long trailing waves near the Lindblad resonance is identical for self-gravitating gaseous and stellar disks. Thus we believe that the angular momentum deposited at the resonance will be carried away by the long trailing wave, just as it is in the gaseous disk. The differences only arise once the long wave has been reflected from corotation as a short wave. In stellar disks the short wave cannot propagate past the Lindblad resonance. Thus the angular momentum carried by the wave will eventually be deposited at the resonance through Landau damping of the short wave.

\section{CONCLUDING REMARKS}

Our major conclusions are described in the Abstract. This section contains some additional comments and a guide to the key equations.

The external torques at the Lindblad and corotation resonances are given by equation (100) or (A10) and by equation (56), (93), or (98), respectively. The angular momentum deposited at a Lindblad resonance is transported away by a long trailing density wave (compare eq. [A10] for the torque to eq. [46] for the angular momentum flux). Short trailing density waves are excited at corotation but do not remove net angular momentum from this 
region. The wave amplitude (eq. [88]) is small for stable disks unless $O(Q-1) \leq O(c / \Omega r) \ll 1$. Strong waves can be excited at corotation in unstable $(Q<1)$ disks, but we doubt that real galactic disks are unstable.

Note that the Lindblad "resonance" is not a true resonance of a fluid disk, but only a region where the disk is strongly coupled to the external potential (i.e., where the WKB wavelengths of the long waves are large; cf. eq. [18]). The external torque is exerted over a distance $\Delta r \sim(G \sigma r)^{1 / 2} / \Omega$. By contrast, the corotation resonance is a true resonance. The torque on the disk there is exerted over a distance $\Delta r \sim c^{2} / G \sigma$.

The perturbations of all physical variables are well behaved at Lindblad resonances, whereas the tangential velocity perturbation has a logarithmic singularity at corotation. This singularity would be removed by the presence of a nonzero shear viscosity.

We thank Drs. J. Bardeen, Y. Y. Lau, C. C. Lin, D. Lynden-Bell, and A. Toomre for helpful discussions. This work was supported by NSF grant AST 76-23281 and NASA grant NGL-05-002-003.

\section{APPENDIX}

The problem addressed here is the validity of the WKB solution of Poisson's equation (cf. eqs. [17], [35], and [58]). This solution is accurate for any disturbance which can be decomposed into Fourier components of one sign of $k$ for which $|k r| \gg 1$.

We have applied equation (17) to the perturbations forced by an external potential near the Lindblad and corotation resonances. The validity of this procedure is open to doubt for two reasons. First, although only trailing waves are excited at each type of resonance, there is also a nonwave part to the perturbation. It is not obvious that the total disturbance can be written as a superposition of Fourier components with positive $k$ (trailing waves). Second, the WKB dispersion relation (eq. [19]) gives $k=0$ for the long wave at the Lindblad resonances. Although this is a formal result, it does cause some concern as the WKB solution of Poisson's equation is valid only if $|k r| \gg 1$. To justify our application of equation (17), we shall demonstrate that the forced disturbances near the Lindblad and corotation resonances may be written as the superposition of Fourier components for which $k r \gg 1$.

\section{a) Lindblad Resonances}

Equation (39) determines the potential perturbation near the resonance. A simple approximate solution of this equation was obtained in $\S$ II. Here we discuss an exact solution, derived by the method of Laplace transform in Goldreich and Tremaine (1978a). Written as a Fourier integral, the solution reads

$$
\varphi_{1}{ }^{D}(x)=-\frac{\alpha \psi}{\beta} \int_{0}^{\infty} d t \exp \left[i\left(t x-\frac{\alpha t^{2}}{2 \beta}+\frac{t^{3}}{3 \beta}\right)\right] .
$$

The integrand has stationary phase at

$$
t_{ \pm}=\frac{1}{2} \alpha\left[1 \pm\left(1-4 \beta x / \alpha^{2}\right)^{1 / 2}\right] .
$$

The contributions to $\varphi_{1}{ }^{D}$ from the regions of stationary phase are of the form

$$
\varphi_{ \pm}(x)=\left(\frac{2 \pi}{|\beta|}\right)^{1 / 2} \frac{|\alpha| \Psi}{\left(\alpha^{2}-4 \beta x\right)^{1 / 4}} \exp \left[i \int^{x} t_{ \pm}(s) d s\right] .
$$

Reference to equations (18) and (19) identifies $\varphi_{ \pm}(x)$ as short and long trailing density waves in the limit $|x| \ll 1$. Note, $t_{-}(x)<0$ for $\beta x<0$ so $\varphi_{-}(x)$ does not exist for $\beta x<0$. This reflects the fact that the long trailing wave exists only between the inner and outer Lindblad resonances. The amplitude of the long trailing wave given by equation (A3) is the same as that given by equation (45) in the limit $x \ll \alpha^{2} / 4 \beta$.

The short trailing wave is present because the long trailing wave excited at the resonance is reflected at $x=$ $\alpha^{2} / 4 \beta$ as a short trailing wave. Since the Laplace transform method gives a global solution of equation (39), we cannot eliminate the short trailing wave as we did in $\S$ II.

The expression for $\varphi_{1}{ }^{D}(x)$ given by equation (A1) satisfies the constraints required for the validity of the WKB solution of Poisson's equation. Only Fourier components with $t \geq 0$ are involved. Although the point of stationary phase which gives rise to the long wave is at $t=0$ for $x=0$, the major contribution to $\varphi_{-}(0)$ comes from $0 \leq$ $t \leq(\beta / \alpha)^{1 / 2}$. Since $O\left[(\beta / \alpha)^{1 / 2}\right] \gg 1$, almost all of $\varphi_{-}(0)$ is generated by spatial frequencies for which $t \gg 1$ or, equivalently, $k r \gg 1$. For $|x| \ll 1$, the nonwave part of $\varphi_{1}(x)$ is largely made up of Fourier components with $t=O\left(|x|^{-1}\right) \gg 1$. Thus the application of the WKB solution of Poisson's equation near the Lindblad resonances is justified.

The behavior of the perturbations of the physical variables near the Lindblad resonances remains to be discussed. This is done most easily in terms of the integral expression for $\varphi_{1}{ }^{D}$ given by equation (A1). Clearly $\varphi_{1}{ }^{D}$ 
and, from Poisson's equation (35), $\eta_{1}$ are well behaved as $x \rightarrow 0$. With the same approximations made in deriving equation (37) from equation (33), the expressions for the perturbed velocities given by equations (12) reduce to

$$
u_{1}=\frac{-i \kappa}{r_{L}|\mathscr{D}| x}\left[\Psi+\frac{d}{d x}\left(\varphi_{1}{ }^{D}+\eta_{1}\right)\right]
$$

and

$$
v_{1}=i \operatorname{sgn}(\mathscr{D}) \frac{\kappa}{2 \Omega} u_{1},
$$

for $|x| \ll 1$. Substituting equation (A1) into equation (A4), we obtain

$$
u_{1}=-\frac{\kappa}{r_{L}|\mathscr{D}|} \Psi \int_{0}^{\infty} d t \exp \left[i\left(x t-\frac{\alpha t^{2}}{2 \beta}+\frac{t^{3}}{3 \beta}\right)\right] \text {. }
$$

Equation (A6) shows that $u_{1}$ is nonsingular at $x=0$. That the same is true for $v_{1}$ is seen from equation (A5). Thus we have proved our assertion in § II. The perturbations of all physical variables are well behaved at the Lindblad resonances.

To calculate the torque exerted on the disk by the external potential in the neighborhood of a Lindblad resonance, we use equation (89) which reads

$$
T=-\pi m \int_{0}^{\infty} d r r \varphi_{1}(r) \operatorname{Im} \sigma_{1}(r)
$$

Using the continuity equation (6) and integrating by parts, we obtain

$$
T=\pi m \operatorname{Im}\left\{\int_{0}^{\infty} d r\left[\frac{m \varphi_{1} \sigma v_{1}}{(m \Omega-\omega)}+i r \sigma u_{1} \frac{d}{d r}\left(\frac{\varphi_{1}}{m \Omega-\omega}\right)\right]\right\}
$$

Near a Lindblad resonance we may rewrite equation (A8) with the aid of equations (4) and (34) in the form

$$
T_{L}=\frac{\pi m \sigma r_{L} \Psi}{\kappa} \operatorname{sgn}(\mathscr{D}) \int_{-\infty}^{\infty} d x \operatorname{Re}\left(u_{1}\right) f(x)
$$

We have treated all quantities except $u_{1}$, the most rapidly varying, as constants. In addition, we have added a weighting factor $f(x)$ which declines monotonically from $f(x)=1$ near the resonance to $f(x) \rightarrow 0$ as $|x| \rightarrow \infty$. Next we substitute for $u_{1}$ using equation (A6). The integral over $x$ is proportional to

$$
g(t)=\int_{-\infty}^{\infty} d x f(x) \exp (i x t)
$$

which peaks sharply at $t=0$ and has a total area of $2 \pi$. Thus

$$
T_{L}=-\frac{\pi m \Psi^{2}}{\mathscr{D}} \int_{0}^{\infty} d t g(t) \exp \left[i\left(\frac{\alpha t^{2}}{2 \beta}+\frac{t^{3}}{3 \beta}\right)\right]=-\frac{\pi^{2} m \sigma^{2} \Psi^{2}}{\mathscr{D}}
$$

This result is in accord with equation (100) (because $\operatorname{sgn}(\mathscr{D})=-l$ ), and it agrees with equation (46) to within a factor sgn (2D). This signum factor arises because the group velocity of long trailing waves has the same sign as $\mathscr{D}$. Thus we have proved that all of the angular momentum deposited at a Lindblad resonance is transported away by a long trailing wave.

\section{b) Corotation Resonance}

Equations (61) and (69) govern the enthalpy perturbation near the corotation resonance. The latter equation is solved by the method of Laplace transform in the following manner. We set

$$
y(v)=\int d z \exp (i z v) g(z)
$$

Substituting equation (A11) into equation (69) and integrating by parts, we obtain

$$
\begin{aligned}
S \exp (-i K v)= & \mid{ }_{-K}^{\infty} \exp (i z v)\left[i\left(z^{2}+b-\frac{v^{2}}{4}\right) g+\frac{v}{4} \frac{d g}{d z}+\frac{i}{4} \frac{d^{2} g}{d z^{2}}\right] \\
& -\int_{-K}^{\infty} d z \exp (i z v) \frac{d}{d z}\left[\frac{1}{4} \frac{d^{2} g}{d z^{2}}+\left(b+z^{2}\right) g\right] .
\end{aligned}
$$


We have chosen the contour of integration to run along the real axis from $-K$ to $+\infty$. Because $v$ has a small positive imaginary part (cf. the second paragraph in $\S$ IV), the boundary terms vanish at $+\infty$.

Equation (A12) is satisfied if

$$
g(-K)=\left.\frac{d g}{d z}\right|_{-K}=0,\left.\quad \frac{d^{2} g}{d z^{2}}\right|_{-K}=i 4 S
$$

and

$$
\frac{d^{2} g}{d z^{2}}+4\left(b+z^{2}\right) g=i 4 S .
$$

Solving equations (A14) subject to the conditions given by equation (A13), we find

$$
g(z)=\frac{S}{2}\left[E(-b, 2 z) \int_{-K}^{2 z} d w E^{*}(-b, 2 w)-E^{*}(-b, 2 z) \int_{-K}^{2 z} E(-b, 2 w)\right] .
$$

Using equations (61), (A11), and (A15), the enthalpy perturbation may be written in the form

$$
\eta_{1}[v(x)]=\frac{\kappa}{(\sigma r)^{1 / 2}} \int_{0}^{\infty} d t \exp [i t v(x)] g(t-K) .
$$

From the asymptotic properties of the parabolic cylinder functions (cf. eq. [71]), it follows that the above expression for $\eta_{1}$ is well defined for all $x$.

The enthalpy perturbation appears as a Fourier integral involving only positive spatial frequencies $(t \geq 0)$. This implies that only trailing disturbances are self-consistent solutions of the linear perturbation equations, at least if the WKB approximation is used to solve Poisson's equation. Thus we confirm the result we obtained in $\S$ IV $b$-namely, only trailing disturbances are forced at corotation in the tight-winding limit. Henceforth, we restrict attention to $K \gg 0$.

The wave parts of $\eta_{1}(x)$ may be evaluated by the method of stationary phase applied to equation (A14). For $|x|>L\left[|v|>\left(2 k_{c} L Q\right)^{1 / 2}\right]$, there is one point of stationary phase for each sign of $x$. Each point corresponds to a short trailing wave which propagates away from corotation. For $\left(Q^{2}-1\right)^{1 / 2} L / Q<|x|<L$, there are two points of stationary phase for each sign of $x$. One of these points is associated with the short trailing wave and the other with the long trailing wave. The latter is weakly excited at $|x|=L$ and propagates toward corotation. It cannot be eliminated from the global solution obtained by Laplace transform. It must be regarded as an artifact of our approximations. This point is discussed in the paragraph following equation (69). The long trailing waves excited at $x= \pm L$ are partially reflected, partially transmitted, and also amplified at corotation (Mark 1976).

Aside from the addition of the long trailing waves, equations (A15) and (A16) describe the perturbation forced near corotation which was more explicitly evaluated in $\S$ III $b$. Equation (A16) demonstrates that this disturbance can be written as a superposition of Fourier components of a single sign. Furthermore, it is easy to show that almost the entire contribution to $\eta_{1}$ comes from short waves, $k r \gg 1$. Note that $g(-K)=0$. Thus the validity of the WKB solution of Poisson's equation near corotation is established.

\section{REFERENCES}

Abramowitz, M., and Stegun, I. A. 1964, Handbook of Mathematical Functions (New York: Dover).

Donner, K. 1978, preprint.

Feldman, S. I., and Lin, C. C. 1973, Stud. Appl. Math., 52,1 .

Goldreich, P., and Tremaine, S. 1978a, Icarus, 34, 240.

. $1978 b, A p . J ., 222,850$.

Kalnajs, A. J. 1972, Ap. Letters, 11, 41.

Kato, S., and Inagaki, S. 1978, preprint.

Lau, Y. Y. 1977, private communication.

Lin, C. C., and Lau, Y. Y. 1975, SIAM J. Appl. Math., 29,

Lin, C. C., and Shu, F. H. 1968, in Astrophysics and General Relativity, ed. M. Chrétien et al. (New York: Gordon \& Breach).

Lynden-Bell, D., and Kalnajs, A. J. 1972, M.N.R.A.S., 157, 1 (LBK).

Mark, J. W. K. 1976, Ap. J., 203, 81.

Shu, F. H. 1970, Ap. J., 160, 99.

Toomre, A. $1969, A p . J ., 158,899$.

‥ 1964, Ap. J., 139, 1217. 1974, Highlights Astr., 3, 457 352 .

Peter Goldreich: Geological and Planetary Sciences, California Institute of Technology, Pasadena, CA 91125 Scott Tremaine: School of Natural Sciences, Institute for Advanced Study, Princeton, NJ 08540 